\title{
The effectiveness of non-pharmacological interventions in improvement of sleep quality among non-remissive cancer patients: A systematic review of randomized trials
}

\author{
Fatmawati*, Luke Budworth \\ School of Psychology, The University of Leeds, Leeds LS3 1BT, The United Kingdom
}

Article history:

Received: 17 October 2016 / Received in revised form: 29 November 2016 / Accepted: 29 November 2016

\begin{abstract}
Statistical results estimated that most of non-remissive cancer patients face sleep problem and experience the symptoms of insomnia throughout and aft er the completion of cancer treatment. The purpose of this review was to compare the effectiveness bet ween several types of non-pharmacological interventions and st andard care or treatment to improve the sleep quality among non-remissive cancerpatients. All randomized st udies focused on non-pharmacological interventions to improve sleep quality among non-remissive cancer patients were included. Thirteen studies were selected with a total of 1,617 participants. The results found that only four interventions were significantly effective to improve sleep quality among non-remissive cancer patients, in cluded cognitive behavioral therapy, relaxation and guided imagery program, self-care behavior education program, and energy and sleep enhancement program.
\end{abstract}

\section{Introduction}

Statistical results estimated that most of non-remissive cancer patients face the problems with sleep and experience the symptoms of insomnia throughout and after the completion of cancer treatment $[1,3,8]$. To reduce these problems, various types of non-pharmacological intervention were performed. However, no further results suggested which of the interventions contributed to significant improvement in sleep quality. A systematic review is needed in order to provide an accurate evidence of each intervention effects as well as to investigate other variables that may contribute to moderate its effects. Consequently, the medical practitioners or oncologists may consider applying the appropriate interventions for improving the sleep quality among the nonremissive cancer patients.

The objective of this review was to investigate the effects of non-pharmacological interventions in improving sleep quality among the non-remis sive cancer patients. A secondary objective was to compare the effects of different types of nonpharmacological interventions in improving sleep quality among the non-remis sive cancer patients.

\section{Methods.}

The following are the methods utilized in this paper.

\footnotetext{
* Corresponding author

Email: ps14ff@leeds.ac.uk
}

\subsection{Eligibility Criteria}

\subsubsection{Types of Studies}

The studies included in this review were randomized trial studies with the sample sizes above fifty (to ensure the statistical power of the results) [14]. These studies were written in English and focused on the non-pharmacological interventions to improve the quality of sleep among nonremissive cancer patients. There were no restrictions for publication date in the studies involved in this review, however, studies published in conference proceedings and theses were exclude.

\subsubsection{Types of Participants}

This review considered the participants with any age who were having cancer or undergoing cancer treatments at the time of the studies, termed as non-remissive cancer patients. The survivors who were in remission from cancer were not included in this review as they experienced less sleep problems compared to the non-remissive cancer patients [1], [2], [3].

\subsubsection{Types of Interventions}

This review included all studies in which nonpharmacological interventions were used as the main 
intervention to improve the sleep quality among nonremissive cancer patients. No specific therapies or interventions were selected as criteria for inclusion in the review.

\subsubsection{Types of Outcome Measures}

Outcome measures in this review are monitored from either subjective or objective measures. The subjective measure was carried out from self-report, whereas the objective measure was taken from the Actigraphy. The primary outcome is sleep quality, including: sleep efficiency (SE), sleep onset latency (SOL), wake after sleep onset (WASO), and total sleep time (TST)

\subsection{Information Sources}

The studies were discovered from screening the electronic databases and only studies used English were included. This search was employed to Medline, Embase, and CINAHL. Additionally, we hand-searched the studies through several journals, including Psycho-Oncology, Cancer Nursing, Oncology Nursing Forum, Palliative and Supportive Care, Patient Education and Counselling, European Journal of Oncology Nursing, Applied Nursing Research, and Cancer Nurs.

\subsection{Search}

We administered the following search terms to search all the studies within the electronic databases.

- Population

Cancer, leukemia, neoplasm, tumor, T-cell, B-cell, etc.

- Intervention

Psychotherapy, supportive expressive group therapy, cognitive therapy, group intervention, self-help group, counselling, expressive writing, etc.

- Outcome

Sleep promotion, sleep help, sleep support, etc.

\subsection{Study Selection}

Firstly, the review authors ran the searches as set up in the search strategy and screened the studies retrieved through the title and the abstract -where the ineligible studies were automatically deleted based on the exclusion criteria. Then, each review author screened half number of total studies remained. In order to measure the inter-rater reliability, each review author shared $12.5 \%$ of his studies screening. When the disagreements were found, the full-text screening was done by using the inclusion criteria to assess the eligibility of the studies to be included in this review. From this evaluation, the inter-rater reliability was 0.96 .

\subsection{Data Collection Process}

The review authors adopted the data extraction sheet based on Cochrane data extraction template, pilot-tested it on thirteen selected studies, and changed it accordingly. The extraction process was done by dividing the included studies into two parts where each part contained half of the number of the thirteen chosen studies. Each part of those divided studies was extracted by one review author, and the extracted data were checked after the process completed. The disagreement between the reviewers resolved through discussion and changes were made appropriately.

\subsection{Data Items}

Information from the selected studies were gained through: (1) general information (including author, title, year, country of origin, stated aim, and study design); (2) characteristics of the participants (including age, sex, type of cancer) as well as the inclusion and exclusion criteria of the participants; (3) intervention information for both main and control intervention (including setting, type of intervention, description of intervention, duration of intervention, and intervention implementer; (4) type of outcome measure (including the scales used to identify the sleep problems and length of follow-up); (5) summary of the results (including the analyses from baseline, post-intervention, and follow-up); and (6) conclusion.

\subsection{Risk of Bias in Individual Studies}

Two review authors assessed the risk of bias within the studies by applying the criteria from the Cochrane risk of bias template as follow:

\subsubsection{Random Sequence Generation}

- Low risk

The studies in this review were ranked as low risk if the authors used the random technique as well as clearly mentioned the process of randomization, and at the end participants were allocated to certain groups based on the randomization procedure.

- Low-medium risk

Low-medium risk was given to the studies which stated the random technique as well as explained the randomization process, but explanation on how the process performed was not clear.

- Medium risk

The studies labelled as medium risk if the authors used the random technique as well as clearly stated the randomization process, but at the end the participants were assigned to certain interventions based on their responses.

- High risk

High risk was rated for the studies which only stated that the participants were randomly assigned to certain groups, but no further explanation on how the process of randomization was given.

\subsubsection{Allocation concealment}

- Low risk

Low risk was labelled for the studies which clearly explained the process to conceal the participant's allocation.

- Medium-high risk

The studies in which the authors mentioned the method to conceal the allocation but not all the study personnel were blinded, were considered as medium-high risk. 
- High risk

Those studies which did not explained the process in concealing the participant's allocation were categorized as high risk.

\subsubsection{Blinding of participants and personnel}

- Low risk

The studies in this review were rated as low risk if the methods to blind the participants and study personnelwere mentioned.

- Medium risk

Medium risk was rated for those studies in which only participants or study personnel were blinded.

- High risk

Those studies that did not mentioned the method to blind the participants and study personnel were labelled as high risk.

\subsubsection{Blinding of outcome assessment}

- Low risk

Low risk was labelled for the studies where the data handlers were blinded from the intervention received by the participants

- Medium risk

Those studies which unclearly explained whether the data handlers were blinded or not, were categorized as medium risk.

- High risk

The high risk was labelled for the studies which did not report the blinding methods used for data handlers.

\subsubsection{Incomplete outcome data}

- Low risk

Low risk was given for those studies which performed the intention to treat analys is if the participant's attrition was found.

- Medium risk

Those studies which did not run the intention to treat analysis and explained certain reasons to support this consideration, were categorized as medium risk.

- High risk

High risk was labelled for those studies which did not perform the intention to treat analysis even the participant's attrition was found and no further reasons to support this consideration.

\subsubsection{Selective reporting}

- Low risk

The studies were labelled as low risk if the outcomes were reported completely as mentioned in the method.

- Medium risk

The studies in which only some results were reported, were categorized as medium risk.

- High risk

High risk was given for those studies in which most of the outcomes were not reported.

\subsection{Summary Measures}

Because the participants, types of intervention, types of outcome measure, and statistical analyses varied across the selected studies, this review focused on describing the results and the limitations found in individual studies.

\section{Results}

\subsection{Study Selection}

A total of 13 studies were eligible to be included in this review. Previously, the search from Medline, PsycINFO, Embase, and CINAHL electronic databases provided as much as 855 citations. Additionally, 10 studies were also identified through hand-searching, thus 865 studies were initially selected. After removing the duplicates, 647 remained. These remaining studies were screened by the review authors based on the title and the abstract. A total of 566 studies were excluded due to some considerations, such as use of pharmacological or invasive intervention only, use of cancer patients in remission or no cancer patients as subjects, lack of clarity on sleep measures, and studies which were classified as theses or conference papers. Then, full-text screening was performed within the remaining 81 studies. Only 13 studies were met the criteria to be involved in this review. Refer to PRISMA flow diagram (Fig. 1) for further detail.

\subsection{Study Characteristics}

\subsubsection{Methods}

All studies included in this review were randomized controlled trial studies published in English. The length of the intervention varied across the studies, with a minimum length of 3 days and maximum of one year. One study did not explicitly mention the duration of the interventions performed [13].

\subsubsection{Participants}

This review included 13 studies with a total of 1617 participants (18 males, 1437 females). The mean age of the participants ranged from 43 to 63 . Most of them were diagnosed with breast cancer, then followed by other types of cancer, such as lung, lymphoma, ovarian, prostate, testicular, bladder, colorectal, bowel, gynecologic, genitourinary, skin, head and neck, gastrointestinal, bone, liver, and brain. Six of the thirteen studies reported the cancer stage among the participants [1-3,5,9,13]. Most of them had stage 2 cancer with a total of 497 patients, then followed by stage 1 (291 patients), stage 3 (132 patients), and stage 4 (46 patients). Furthermore, only two studies in this review reported the length of insomnia experienced by the participants [7-8]. One study stated that the patients in the intervention group had the average duration of 30 months, whereas the no-intervention control group experienced the average duration of 27 months [7]. However, another study reported longer duration where the patients in first intervention group experienced 6.74 years in average and the patients in second intervention group experienced 6.67 years in average [8]. 
In addition, from those included studies, there were two studies that mentioned the previous cancer treatments taken by the participants [4], [8], including surgery, chemotherapy, radiation therapy, and hormonal therapy. Most of the patients underwent the chemotherapy, as much as 83 patients.

\subsubsection{Interventions}

From all selected studies, six studies were conducted in the USA and two studies were identified from the UK, whereas the rest of five studies were discovered from Denmark, Israel, Canada, Netherland, and Taiwan. Five studies in this review operated cognitive behavioral therapy for intervention group $[4,6-8,11]$ and the remaining studies performed several types of treatment, including mindfulness-based stress reduction program [1], behavioral therapy-individualized sleep promotion time [3], energy and sleep enhancement [2], exercise therapy [5], stress management training [9], massage therapy [10], writing exercise [12], and self-care behavior education [13]. Furthermore, control group within the six studies received certain intervention -for example, nutritional education, relaxation and guided imagery, and healthy eating control, whereas the rest control group in the remaining studies received no intervention or treatment as usual.

\subsubsection{Outcomes}

From all studies, the outcome assessed was sleep quality, including sleep efficiency (SE), sleep onset latency (SOL), wake after sleep onset (WASO), and total sleep time (TST). Besides, some of the outcome measures also assessed the sleep disturbance and sleeping medication use. These outcomes were observed through objective (Actigraph) as well as subjective measures (self-report). There were several subjective measures used in those selected studies, involving Medical Outcome Study Sleep Scale, The Pittsburgh Sleep Quality Index, Morin Sleep Diary, The Mini Sleep Questionnaire, The General Sleep Disturbance Scale, A 10 Day Sleep Diary, The Insomnia Severity Index, The Subjective Sleep Quality Scale, The Richard-Campbell Sleep Questionnaire, and Self-Care Diary. In addition, there were four studies used Actigraph in order to measure the sleep quality $[2,3,6,8]$.

\subsection{Risk of Bias within Studies}

\subsubsection{Random Sequence Generation}

Within thirteen selected studies, nine studies were labelled as low risk since they completely reported the randomization process [1,5-12]. Most of them used a computer based random assignment program in order to minimize the random error. There was one study involved in medium-low risk as the authors did not clearly mention on how the random assignments were done [2]. One study by Berger et al. [3] was rated as medium risk because the allocation of participants into the intervention group was determined by their baseline responses. Lastly, high risk was given for two studies because the randomization sequence was not reported, each of them was conducted by Cohen and Fried [4] and Williams and Schreir [13]. Thus, it can be concluded that most of the selected studies in this review had a good method in generating the allocation sequence.

\subsubsection{Allocation Concealment}

In concealing the allocation, there were five studies categorized as low risk as the method used in allocation concealment was clearly reported, for example by using the sequentially numbered envelopes [2,5,8,11-12]. One study by Jane et al. [10] was classified as medium-high risk because not all the study personnel were blinded from the allocation concealment. The seven remaining studies were labelled as high risk because the authors did not explain the technique to conceal the allocation $[1,3-4,6-7,9,13]$. Hence, it can be said that most of studies in this review had a poor technique in concealing the allocation sequence.

\subsubsection{Blinding of Participants and Personnel}

There were only two studies in this review categorized as low risk in blinding the participants and personnel $[5,8]$. Within these studies, both participants and study personnel were blinded to which intervention the participants assigned commonly known as double-blind method. Then, five studies were labelled as medium risk since only participants or study personnel was blinded -termed as single blind method [2$4,9,12]$. The studies that did not clearly mentioned the method used to blind the participants and study personnel were classified as high risk. This label was given for six studies [1,6-7,10-11,13]. From the explanations above, it can be concluded that most of the studies involved in this review did not completely describe the method to blind the participants and study personnel.

\subsubsection{Blinding of Outcome Assessment}

Two studies in this review were rated as low risk because the authors clearly mentioned the technique used to blind the data handlers from awareness of which intervention the participants received [2,4]. Furthermore, only study by Jane et al. [10] was labelled as medium risk as they did not explicitly state whether the data handlers were blinded or not. High risk was given for the remaining ten studies because the technique used to blind the data handlers was not reported [1,3,5-9,1113]. Thus, it can be said that most of the studies in this review did not applied a certain technique to blind the outcome assessors from which intervention the participants allocated in.

\subsubsection{Incomplete Outcome Data}

Ten studies in this review were categorized as low risk [1,3,5-6,8-13]. This label was given because those studies performed the intention to treat analysis to minimize to bias from participant attrition. However, if this analysis was not reported, the study will be classified into high risk and this label was marked into two studies [4,7]. Only one study in this review was ranked as medium risk [2], the consideration was because this study did not perform the intention to treat analysis among 16 dropped out participants and just excluded them due to the poor health responses during the baseline assessment. To conclude, most of included studies in this 
review had a complete outcome data as the intention to treat
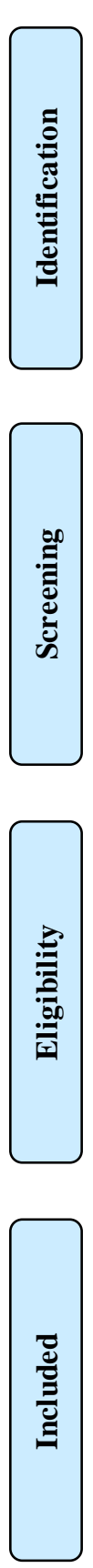

$$
(n=647)
$$

screened

$(\mathrm{n}=647)$

analysis was performed to avoid the participant attrition bias.

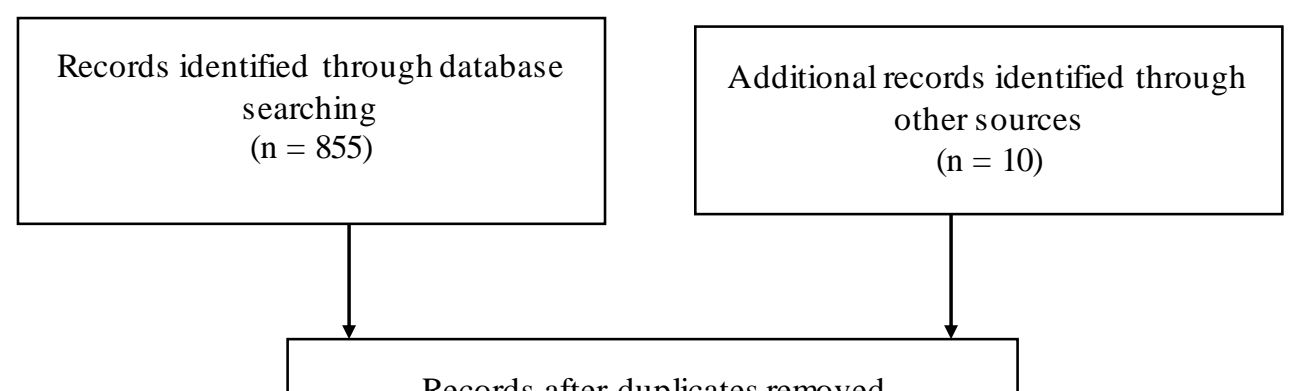

Records after duplicates removed

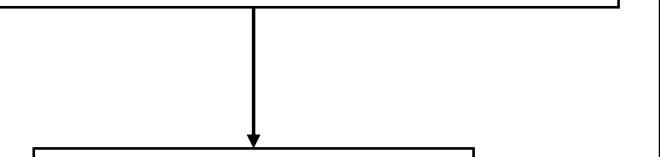

Records excluded

$$
(n=566)
$$

- Used a pharmacological and/or invasive intervention only

- Contained cancer patients in remis sion/no cancer patients

- Did not include sleep measures

- Theses/conference papers

- Unfound duplicates

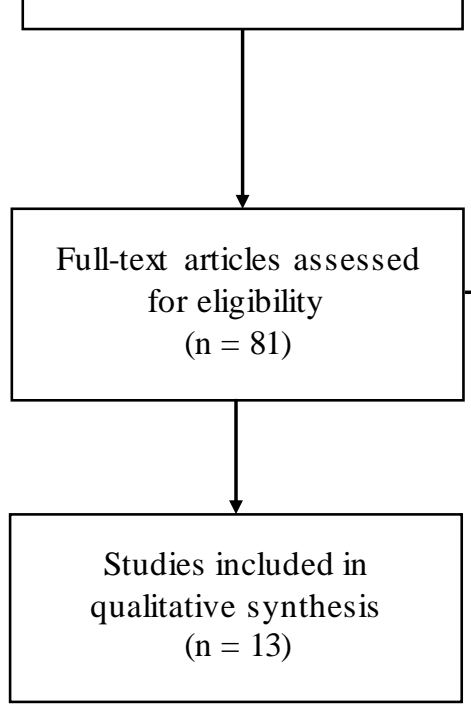

Full-text articles excluded,

$$
(n=68)
$$

- Sample size less than 50

- Non-randomised studies

- Unfound conference papers

- 'Quasi-duplicates'

Fig. 1. Flow diagram of study selection

\subsubsection{Selective Reporting}

For selective reporting bias, ten studies had a low risk level since they completely reported all the results [2-4,6,813]. Medium risk was given for study conducted by Andersen et al. [1] due to incomplete results reported, such as no outcome informed for the analysis of group differences in sleep change between baseline and follow-up. The study in which most of the important results were not reported, were classified into high risk -for example, no data for follow-up results and no mean scores for each outcome measure. This label was given for studies by Dodd et al. [5] and Fleming et al. [7]. From these descriptions, it can be concluded that most of the studies selected in this review reported a complete result for each analysis mentioned in the study method.

\subsection{Results of Individual Studies}

\subsubsection{Study 1}

Andersen et al. [1] performed the Mindfulness-Based Stress Reduction (MBSR) program to examine its effects towards the sleep quality among 168 breast cancer patients. The Medical Outcome Study Sleep Scale (MOS-Sleep) was used in order to gather the data. This intervention was completed in eight weeks with two-hour session on every week. Then, after 6 months and 12 month of intervention, the follow-up assessments were carried out. The analyses 
revealed that no significance mean changes in sleep scale scores from baseline to post-treatment and follow-up. It suggested the MBSR did not contribute to the long-term effect of sleep quality among the breast cancer patients.

\subsubsection{Study 2}

Two interventions were performed by Barsevick et al. [2] in order to reduce the sleep disturbance among 292 cancer patients who underwent the chemotherapy. The intervention group received the Energy and Sleep Enhancement (EASE) program, whereas the control group was given the Nutritional Education and Dietary Improvement Guidance. Through the Octagonal Basic Motionlogger Actigraph (objective sleep measure), the significant result was found in WASO score ( $p$ $<0.05$ ), where the intervention group showed greater improvement than control group from baseline to follow-up. In addition, night-time awakenings also significantly reduced in both group from the baseline to follow-up $(p<0.05)$ where the greater reduction was also identified in intervention group.

\subsubsection{Study 3}

In order to improve the sleep quality among 173 breast cancer patients who received the adjuvant chemotherapy treatment, Berger et al. [3] compared the effectiveness of two interventions, including the behavioral therapy sleep intervention -termed as Individualized Sleep Promotion Plan (ISPP) - and Healthy Eating Control (HEC) intervention. The results from the Pittburgh Sleep Quality Index (PSQI) revealed that both groups were categorized as poor sleeper because the mean scores were above five on each group. However, over a year follow-up, the ISPP group showed significant improvement in sleep quality compared to the HEC group $(p<0.001)$. This indicated that behavioral therapy was effective in improving the long-term sleep quality among the breast cancer patients.

\subsubsection{Study 4}

The study compared the effectiveness of relaxation and guided imagery training (RGI) and cognitive-behavioral group therapy (CBT) and was performed by Cohen and Fried [4] in order to reduce the sleep difficulties among the cancer patients. Results from the Mini Sleep Questionnaire (MSQ) revealed that RGI was more effective than BCT in reducing sleep difficulties. This captured from mean difference in each group during baseline until 4-month follow-up, where the RGI got higher mean difference of $11.57(\mathrm{SD}=-0.1)$ compared to BCT $(\mathrm{M}=5.36, \mathrm{SD}=3.73)$.

\subsubsection{Study 5}

Dodd et al. [5] did an exercise prescription intervention in women during and after chemotherapy, named as the Pro-self: Fatigue Control Program. The General Sleep Disturbance Scale (GSDS) was used to evaluate the aspects of sleep disturbance, including quality and quantity of sleep, sleep latency, waking up during sleep, daytime sleepiness, and medication use. The intervention was implemented into two different groups in which the first group (EE) received the intervention a week before second chemotherapy session, while the second group (CE) was delivered the intervention after completing the cancer treatments. The results from multilevel regression analyses showed there were no significance change in sleep disturbances over the time as well as between the groups. This indicated that exercise intervention was not effective to reduce sleep disturbances among cancer women.

\subsubsection{Study 6}

The study conducted by Espie et al. [6] aimed to examine the effectiveness of cognitive behavioral therapy to reduce the symptom of insomnia among 100 cancer patients. Sleep diary and Actigraph were employed in order to measure WASO, SOL, TST, and SE. The results after six-month follow-up indicated there was a statistically significant reduction in WASO, SOL, and SE $(p<0.001)$, however no significance change was found in TST. This revealed that cognitive behavioral therapy was a clinically effective approach to reduce the insomnia occurred among the cancer patients.

\subsubsection{Study 7}

In order to determine the levels of sleep disturbance among the cancer patients, Fleming et al. [7] applied the cognitive behavior therapy for insomnia (CBT-I) by using a 10-day sleep diary as the purpose of gaining the data. The results from pre- to post-treatment data showed there was a significant reduction of sleep disturbances among CBT-I group and control group ( $p<0.001$ ), where the CBT-I group reduced higher than control group with amount of 52\% and $17.5 \%$, respectively. This meant that CBT-I had a good impact in decreasing the insomnia among the cancer patients.

\subsubsection{Study 8}

The study examined the effectiveness of mindfulnessbased stress reduction (MBSR) program and cognitive behavioral therapy in reduction of insomnia among cancer patients was performed by Garland et al. [8]. Both subjective and objective sleep measure were administered in order to gather the data about sleep quality. The results from sleep diaries indicated that at follow-up assessment, SOL and SE improved significantly in CBT-I group $(p<0.001)$, whereas WASO and TST improved significantly in MBSR group ( $p<$ 0.001). However, the Actigraph detected that SOL, SE, and WASO progressed significantly in CBT-I group $(p<0.05)$, and only TST progressed significantly in both groups $(p<$ 0.001). Those results revealed that CBT-I contributed to the greater improvement for insomnia among cancer patients.

\subsubsection{Study 9}

Garssen et al. [9] conducted a study to evaluate the quality of sleep among breast cancer surgery patients by implementing the stress management training program (SMT). This intervention was applied within patients who had stage one cancer. The Subjective Sleep Quality Scale was employed to collect the information about sleep quality and sleep medication consumption. The follow-up data was gathered after one month of intervention and the results showed that no significance mean differences found between SMT group and control group over the time. This implied that SMT intervention did not provide an effective contribution towards 
the improvement of sleep quality among breast cancer surgery patients.

\subsubsection{Study 10}

The study proposed by Jane et al. [10] aimed to compare the efficacy of Massage Therapy (MT) and Social Attention program (SA) towards the 72 Taiwanese cancer patients. The Richard-Campbell Sleep Questionnaire with visual analogue form was functioned in order to measure the sleep quality level among the participants. The results from the analyses presented that there was no significant improvement of sleep quality in both MT and SA group. This inferred that those interventions were less appropriate to be applied among cancer patients to improve the sleep quality.

\subsubsection{Study 11}

A study investigated the sleep disturbance among cancer patients who received advanced cancer treatments was proposed by Kwekkeboom et al. [11]. This study implemented the Patient-Controlled Cognitive Behavior intervention (PC$\mathrm{CB}$ ) by employing the numeric rating scale and the Pittsburgh Sleep Quality Index (PSQI) to collect the data. The results obtained that although the reduction towards sleep disturbance was gained, but there was no statistical difference found between the intervention and control group $(p=0.47)$. This suggested that PC-CB had less impact on sleep disturbance among advanced cancer treatment patients.

\subsubsection{Study 12}

The emotionally expressive writing was applied by Low et al. [12] in order to measure the sleep quality among women with metastatic breast cancer. The Pittsburgh Sleep Quality Index (PSQI) was used for gathering the data. The baseline results showed that $74 \%$ of the participants experienced the significant sleep disturbances. During the follow-up as sessment, the intervention group demonstrated greater sleep disturbances than control group with the mean score of 7.1 $(\mathrm{SD}=0.51)$ and $6.6(\mathrm{SD}=0.51)$, respectively. This occurred because most of patients in this group had been living with cancer in longer period, thus they exhibited more negative thoughts regarding the experience towards cancer which then impacted on their sleep. This implied that emotionally expressive writing was ineffective to be implemented among long period cancer patients.

\subsubsection{Study 13}

In order to reduce the side-effect of sleep problems among breast cancer women who underwent the chemotherapy, the benefit of self-care behavior education was examined by Williams and Schreier [13]. The data about sleep disturbances were gained through a Self-Care Diary (SCD) after 1 month and 3 month of intervention. The results suggested that educational intervention helped the patients in managing the sleep difficulty and most of them were able to use wider variety of self-care behavior for reducing the sleep problem symptoms. Hence, this revealed that self-care behavior education was suitable to be applied among breast cancer patients.

\section{Discussion}

\subsection{Summary of Evidence}

From the explanation presented above, we identified that there were four non-pharmacological interventions significantly contributed to the improvement of sleep quality among the non-remissive cancer patients. Those interventions include cognitive behavioral therapy [3,6-8], relaxation and guided imagery program [4], self-care behavior education program [13], and energy and sleep enhancement program [2]. Besides, there were also some non-pharmacological interventions which contributed to less improvement, such as mindfulness-based stress reduction program [1], home-based exercise program [5], stress management training [9], and massage therapy [10] as well as emotionally expressive writing therapy [12].

Cognitive behavioral therapy was widely assessed to be an effective intervention in reducing sleep difficulties among cancer patients because it combined the adequate components targeted to modify the cognitive disturbances arisen from the stress which commonly occurred during the cancer treatments [6-8]. Furthermore, relaxation and guided imagery program was also effective to be implemented in increasing sleep quality. This intervention associated with relaxation technique involved in the treatment process which helped the patients to manage the side-effects due to cancer treatments. [4]. The self-care behavior education program contributed to significant result in enhancing sleep quality because the education received allowed the cancer patients to apply wider variety of self-care behavior, thus the way they managed the sleep problems would be more efficient [13]. In addition, the energy and sleep enhancement program had the same procedure with educational intervention because it provided the education about sleep disturbances, but the intervention process then elaborated comprehensively as the coping and appraisal phases were also included in the intervention session, so the sleep disturbances would reduce effectively [2].

\subsection{Limitations}

Some limitations were found within the selected studies in this review. One of them was due to the bias in allocation concealment where seven studies were rated as high risk because the method used to conceal the allocation sequence was not reported. Moreover, the bias in blinding the study personnelas well as outcome assessors were also high as most of the studies did not explicitly stated the method performed. Also, only few studies operated the Actigraph while the others preferred to use self-report. The use of objective sleep measure will be very helpful for providing more actual results because sometimes the participants face the difficulties to complete the questionnaire. Besides, the absence of nointervention control group also contributed to the limitation of this review because the results obtained could not be compared with the participants who did not receive any interventions. 


\section{Conclusion}

\subsection{Implications for Practice}

The results of this review were based on thirteen included studies with various types of cancer. The nonpharmacological interventions have been implemented among the non-remissive cancer patients in order to improve the quality of sleep and the effects of those interventions varied from one to another. The data from individual studies also revealed that those interventions not only reduced the sleep disturbances, but also other kind of psychological symptoms, such as fatigue, mood, stress, anxiety, locus of control, and quality of life.

\subsection{Implicationsfor Research}

The results from individual studies indicated that several non-pharmacological interventions were effective to improve sleep quality among non-remissive cancer patients. However, more intervention techniques are required in order to provide more comprehensive evidence. In addition, it is also important to conduct the qualitative study to enlarge the knowledge regarding the patients' experience towards sleep problems. The non-randomized study might be also considered as it can help reducing the attrition of participants caused by the lack adherence to intervention condition. Lastly, the power analysis also need to be included in future studies, thus the number of recruited participants are adequate.

\section{References}

1. S. R. Andersen, H. Wurtzen, M. Steding-Jessen, J. Christensen, K. K. Andersen, H. Flyger, C. Mitchelmore, C. Johansen an d S. O. Dalt on, Effect of mindfulness-based stress reduction on sleep quality: Results of a randomized trial among Danish breast cancer patients, Acta Oncol. 52 (2013) 336-344

2. A. Barsevick, S. L. Beck, W. N. Dudley, B. Wong, A. M. Berger, K. Whitmer, T. Newhall, S. Brown and K. Stewart, Efficacy of an intervention for fatigue and sleep disturbance during cancer chemotherapy, J. Pain Symptom. Manage. 40(2010) 200-2 16.

3. A. M. Berger, B. R. Kuhn, L. A. Farr, S. G. Von Essen, J. Chamberlain, J. C. Lynch and S. Agrawal, One-year outcomes of a behavio ral the rapy intervention trial on sleep quality and cancer-related fatigue. J. Clin . Oncol. 27 (2009) 6033-6040.

4. M. Cohen and G. Fried, Comparing relaxation training and co gn itive behavioral group therapy for women with breast cancer, Res. Soc. Work Pract. 17 (2007)313-323.

5. M. J. Dodd, M. H. Cho, C. Miaskowski, P. L. Painter, S. M. Paul, B. A. Cooper, J. Duda, J. Krasnoff and K. A. Bank, A randomized controlled trial of home-based exercise for cancer-related fatigue in women during and after chemotherapy with or without radiation therapy. Cancer Nurs. 33 (2010) 245-257.

6. C. A. Espie, L. Fleming, J. Cassidy, L. Samuel, L. M. Taylor, C. A. White, N. J. Douglas, H. M. Engleman, H. L. Kelly and J. Paul, Randomized controlled clinical effectiveness trial of cognitive b e havior therapy compared with treatment as usual for persistent insomnia in patients with cancer, J. Clin. Oncol. 26(2008)4651-4658.

7. L. Fleming, K. Randell, C. J. Harvey and C. A. Espie, Does cognitive behavior therapy for insomnia reduce clinical levels offatigue, anxiety and depression in cancer patients?, Psycho-Oncology 23(6) (2014)679684.

8. S. N. Garland, L. E. Carlson, A. J. Stephens, M. C. Antle, C. Samuels and T. S. Campbell, Mindfulness-based stress reduction compared with cognitive behavioral therapy for the treatment of in somnia comorbid with cancer: A randomized, partially blinded, noninferiority trial, J. Clin. Oncol. 32(5) (2014) 449-457.

9. B. Garssen, M. F. Boomsma, E. De Jager Meezenbroek, T. Porsild, J. Berkhof, M. Berbee, A. Visser, S. Meijer and R. H. J. Beelen, Stress management training for breast cancer surgery patients, PsychoOncology 22(3) (2013) 572-580.

10. S. W. Jane, S. L. Chen, D. J. Wilkie, Y. C. Lin, S. W. Foreman, R. D. Beaton, J. Y. Fan, M. Y. Lu, Y. Y. Wang, Y. H. Lin and M. N. Liao, Effects of massage on pain, mood status, relaxation, and sleep in Taiwanese patients with metastatic bone pain: A randomized clinical trial, Pain 152(10)(2011)2432-2442.

11. K. L. Kwekkeboom, K. Abbott-Anderson, C. Cherwin, R. Roiland, R. C. Serlin and S. E. Ward, Pilot randomized controlled trial of a patientcontrolled cognitive-behavioral intervention for the pain, fatigue, and sleep disturbance symptom cluster in cancer, J. Pain Symptom. Manage. 44(6) (2012) 810-822.

12. C. A. Low, A. L. Stanton, J. E. Bower and L. Gyllenhammer, A randomized controlled trial of emotionally expressive writing for women with metastatic breast cancer, Health Psychol. 29(4) (2010) 460-466.

13. S. A. Williams and A. M. Schreier, The role of education in m an aging fatigue, anxiety, and sleep disorders in women undergoing chemotherapy for breast cancer, Appl. Nurs. Res. 18(3)(2005) 138-147.

14. H. White, S. Sabarwal, and T. de Hoop, Randomized Controlled Trials (RCTs): Methodological Briefs-Impact Evaluation, UNICEF 7 (2014) 1 15 . 